\title{
Leukostasis-Related Fatal Cardiopulmonary Arrest as Initial Chronic Myeloid Leukemia Presentation
}

\author{
Konstantinos Katogiannis ${ }^{\mathrm{a}}$, Ignatios Ikonomidis ${ }^{\mathrm{a}}$, Fotios Panou ${ }^{\mathrm{a}}$, Andreas Katsimardos ${ }^{\mathrm{a}}$, \\ Aspasia Divane ${ }^{\mathrm{b}}$, Argyrios Tsantes ${ }^{\mathrm{c}}$, Efstathios Iliodromitis ${ }^{\mathrm{a}}$, \\ Elias Kyriakou ${ }^{\mathrm{c}, \mathrm{d}}$
}

\begin{abstract}
Chronic myeloid leukemia (CML) is a BCR-ABL1-positive myeloproliferative neoplasm (MPN), which is usually diagnosed in chronic phase (CP-CML) and is considered curable in the era of tyrosine kinase inhibitors (TKIs). Leukostasis is an urgent complication more often of acute and less often of chronic leukemias. Regarding CML, leukostatic symptoms are uncommon in $\mathrm{CP}$ and in newly diagnosed patients, while being more often in advanced disease stages (accelerated or blastic phase). Leukostasis in CML is mostly presented with neurologic or respiratory symptoms. We present here a case of newly diagnosed CML in CP, which initially presented with leukostasis-related fatal cardiopulmonary arrest in a 59-year-old woman.
\end{abstract}

Keywords: Chronic phase CML; Leukostasis; Cardiopulmonary arrest

\section{Introduction}

Chronic myeloid leukemia (CML) is a myeloproliferative neoplasm (MPN) that accounts for $15-20 \%$ of all cases of leukemia in adults. CML annual incidence is $1.0-1.5$ per 100,000 persons, while the median age of onset is 50 - 60 years. Signs and symptoms at presentation result mainly from anemia and splenomegaly and may include fatigue, lethargy, weight loss, sweats, malaise and left upper quadrant fullness or pain. Rare manifestations include bleeding due to thrombocytopenia and/ or platelet dysfunction, thrombosis due to thrombocytosis and/ or marked leukocytosis and gouty arthritis due to elevated uric

Manuscript submitted December 20, 2017, accepted January 15, 2018

a2nd University Department of Cardiology, "Attikon” University Hospital, Athens, Greece

bPrivate Diagnostic Laboratory, "LIFE CODE Medical Ltd", Athens, Greece 'University Department of Laboratory Haematology, Haemostasis and Blood Bank, "Attikon" University Hospital, Athens, Greece

${ }^{\mathrm{d} C}$ Corresponding Author: Elias S. Kyriakou, University Department of Laboratory Haematology, Haemostasis and Blood Bank, "Attikon" University Hospital, 1 Rimini Str, PC 12462, Chaidari, Athens, Greece.

Email:kyriakoue72@gmail.com

doi: https://doi.org/10.14740/jmc2993w acid levels. In developed countries, about $50 \%$ of patients are initially asymptomatic and are diagnosed as a consequence of blood tests performed for unrelated reasons, showing profound leukocytosis with left shift of differentiation. More than $90 \%$ of CML patients are diagnosed in a relatively early CML stage, known as the chronic phase (CP), while the rest $10 \%$ are diagnosed in advanced stage. The latter is divided into an initial accelerated phase (AP), during which patients may still respond to treatment for some months or years and a subsequent more aggressive blastic phase (BP), with a median survival of approximately 6 months. AP might be insidious or present with worsening anemia, splenomegaly and organ infiltration, while $\mathrm{BP}$ presents as an acute leukemia [1-3].

CML is characterized by a consistent cytogenetic abnormality (the $\mathrm{t}[9 ; 22][\mathrm{q} 34 ; \mathrm{q} 11]$ chromosomal translocation, so called "Philadelphia chromosome") and the presence of the BCR-ABL1 fusion gene. The BCR-ABL1 fusion gene encodes the oncoprotein BCR-ABL1 (p210) with a constitutive active tyrosine kinase activity, which is the primary cause of the $\mathrm{CP}$ of CML. Diagnostic criteria of CP-CML include leucocytosis with or without thrombocytosis, left shift of WBC differentiation, basophils $<20 \%$, splenomegaly, $<10 \%$ myeloblasts in peripheral blood or bone marrow and Philadelphia chromosome/BCR-ABL1 fusion gene in bone marrow or blood detected by cytogenetic/PCR analysis. Diagnostic criteria of AP-CML include $10-19 \%$ myeloblasts in bone marrow or blood, basophils $>20 \%$, platelets $<100,000 / \mu \mathrm{L}$ unrelated to therapy or platelets $>1,000,000 / \mu \mathrm{L}$ unresponsive to therapy, cytogenetic evolution with new cytogenetic abnormalities and increasing splenomegaly or white blood cell count unresponsive to therapy, while diagnostic criteria of BP-CML include > $20 \%$ myeloblasts in bone marrow or blood $[1,4,5]$.

The discovery in 1996 that BCR-ABL1 kinase activity could be pharmacologically inactivated by imatinib mesylate, introduced this first generation tyrosine kinase inhibitor (TKI) as the initial oral targeted treatment for newly diagnosed CML patients, replacing interferon, Ara-C and hydroxycarbamide. Three second generation inhibitors and one third generation inhibitor are today available and potent to surmount primary or secondary resistance to imatinib. Of the second generation drugs, nilotinib and dasatinib are licensed for patients with CPCML even as first-line treatment, while bosutinib is currently licensed for CML patients resistant or refractory to first-line drugs. The third generation inhibitor ponatinib is licensed for CML patients who either have a T315I mutation or fail to re- 


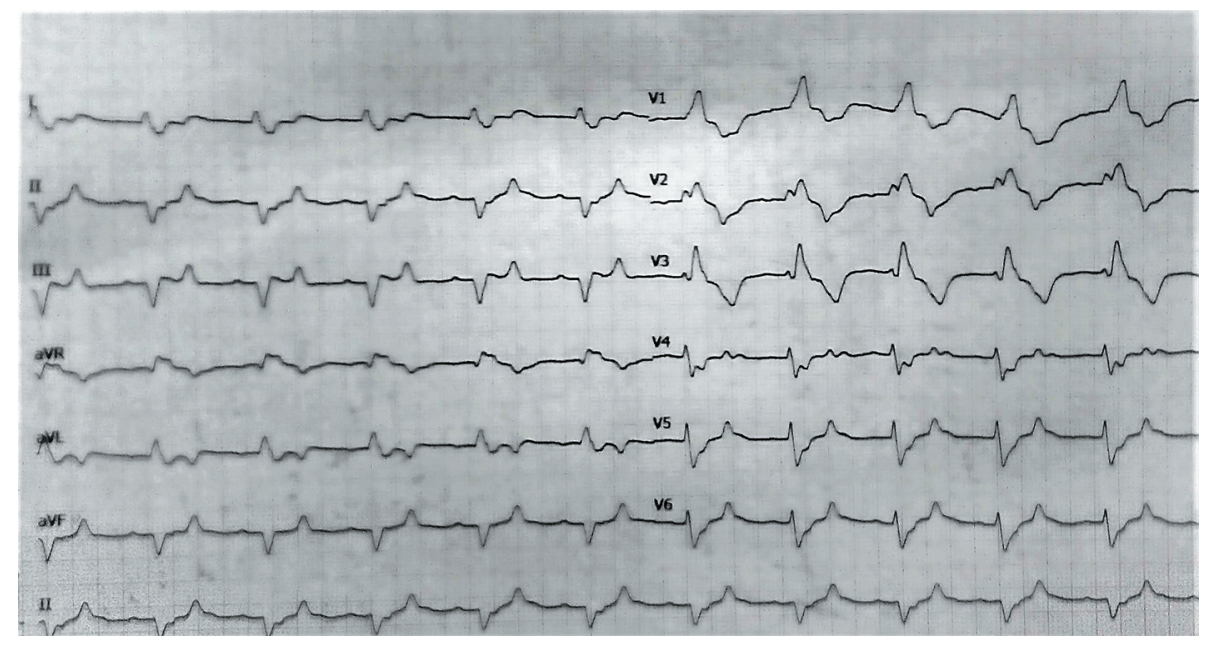

Figure 1. Electrocardiogram with sinus rhythm, RBBB pattern and right axis deviation.

spond to any of the other currently approved TKIs. Allo-SCT is being restricted to patients who have failed multiple lines of TKIs, or harbor a T315I mutation and are either ineligible for or have failed ponatinib. Sokal score developed in 1984 for patients treated with busulfan, Hasford score developed in 1998 for patients treated with IFN and EUTOS score developed in 2011 for patients treated with TKIs, have all been confirmed to be useful in stratifying patients into good-, intermediate-, and poor-risk categories, thus promoting decision-making process regarding appropriate treatment options. European Leukemia Net (ELN) has recently defined criteria of CML molecular response (MR) assessment by RQ-PCR at 3, 6 and 12 months. MR reflects the reduction of $\mathrm{BCR}-\mathrm{ABL} 1$ fusion transcripts, while optimal response is defined as BCR-ABL1 transcript levels of less than $10 \%$ at 3 months, less than $1 \%$ at 6 months, and less than $0.1 \%$ from 12 months onwards $[1,2,4]$.

Leukostasis is one of the serious complications of CML, more often in BP, and is characterized by partial or total occlusion of microcirculation by aggregation of leukemic cells and thrombi leading mainly to respiratory or neurologic symptoms. The so-called "leukostatic symptoms" are uncommon nowadays in newly diagnosed CML patients in $\mathrm{CP}$ or AP, despite WBC counts exceeding $100 \times 10^{9} / \mathrm{L}[3,6]$.

We present here a case of a 59-year-old female who initially manifested cardiopulmonary arrest due to severe leukostasis originating from newly diagnosed -not in BP - CML and died before receiving any kind of specific treatment.

\section{Case Report}

A 59-year-old woman was admitted to our hospital's emergency department because of acute dyspnea and altered mental status. Her dyspnea had occurred 2 days before and had been gradually aggravated, along with bilateral ankle swelling.

Except from being a smoker, her medical history included only major depression due to her husband's recent death, which was treated with escitalopram.

Upon her arrival at the emergency department, her clinical condition was critical. She was almost pulseless and gasping with a respiratory frequency of 5 breaths per minute. Cardiopulmonary resuscitation started immediately, the patient was intubated and mechanical ventilation was applied. After providing chest compressions, signs of circulation appeared shortly after and central arteries became palpable. After circulation recovery, a 12-lead electrocardiogram (Fig. 1) revealed sinus rhythm, with an RBBB pattern and a frequency of 65 beats per minute. The QRS was approximately $140 \mathrm{~ms}$ wide and a rightaxis deviation was noted. Her blood pressure was $70 / 40 \mathrm{~mm}$ $\mathrm{Hg}$ and the patient was supported with intravenous fluids $(\mathrm{N} / \mathrm{S}$ $0.9 \%)$ at a rate of $200 \mathrm{~mL} / \mathrm{h}$ and nor-adrenaline solution $(8 \mathrm{mg}$ in $100 \mathrm{~mL} \mathrm{~N} / \mathrm{S} 0.9 \%$ ) at a rate of $20 \mathrm{~mL} / \mathrm{h}$. A 3-lumen central venous catheter was placed in the right femoral vein. Arterial blood gas revealed severe mixed (respiratory, metabolic and lactic) acidosis $\left(\mathrm{pH}=6.97, \mathrm{pO}_{2}=48 \mathrm{~mm} \mathrm{Hg}, \mathrm{pCO}_{2}=85 \mathrm{~mm}\right.$ $\left.\mathrm{Hg}, \mathrm{HCO}_{3}^{-}=18 \mathrm{mmol} / \mathrm{L}, \mathrm{Lac}=10.5 \mathrm{mmol} / \mathrm{L}\right)$. After her blood pressure had risen up to $100 / 60 \mathrm{~mm} \mathrm{Hg}$, intravenous solution of bicarbonate $8.4 \%$ was administered, along with $5 \mathrm{mg}$ inhaled salbutamol. Due to severe hyperkalemia, $60 \mathrm{mg}$ furosemide and $250 \mathrm{~mL} \mathrm{D/W} \mathrm{10 \%} \mathrm{solution} \mathrm{enriched} \mathrm{with} 10 \mathrm{IU}$ crystalline insulin were given intravenously and a fleet enema of polystyrene sulfonate was applied, while dialysis treatment was planned. Subsequently, auscultation of thorax divulged clear $\mathrm{S}_{1}-\mathrm{S}_{2}$ heart sounds without murmurs and normal respiratory whispering at both lung fields. Abdomen was dilated but painless with normal intestinal sounds. There was a palpable liver and a palpable spleen both $5 \mathrm{~cm}$ below the right and the left pleural arc, respectively. Edema was present at both legs below the knees with an imprint on pulsation. Jugular veins were bilaterally distended up to $10 \mathrm{~cm}$, while upper and lower extremities were cold and pale. Chest X-ray illustrated an increased cardiopulmonary index, bilateral pleural effusion and cotton-wool filtrates at both lung fields (Fig. 2), while the role of point of care echocardiography was critical (Fig. 3), since it revealed normal left ventricle dimensions, satisfying ejection fraction, normal left atrial dimension, absence of pericardial fluid, appropriate structure and function of pulmonary, aortic and mitral valve, but severely dilated right ventricle (almost 54 


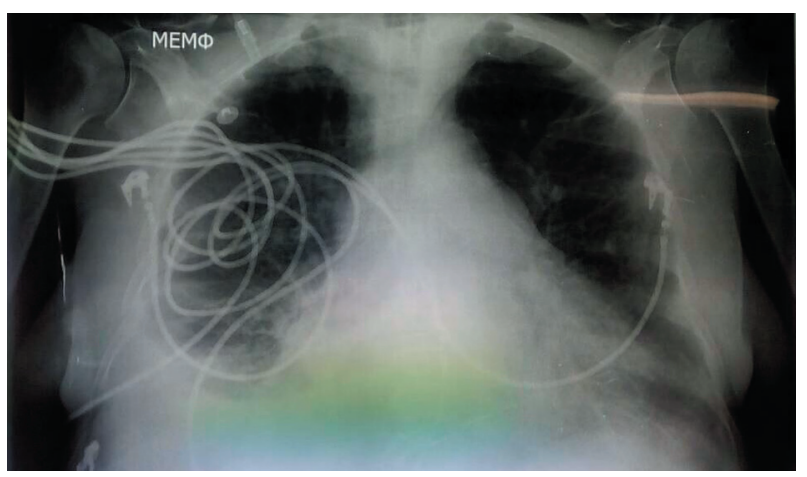

Figure 2. Patient's chest $\mathrm{X}$-ray with increased cardiopulmonary index and cotton-wool infiltrates.

$\mathrm{mm}$ from the meditation of four-chamber view) and presence of D-sign at both diastole and systole, along with right atrium dilation and severe tricuspid valve regurgitation (systolic flow reversal in hepatic vein) with a maximum regurgitation velocity of $2.8 \mathrm{~m} / \mathrm{s}$. Besides, inferior vena cava was found dilated (27 $\mathrm{mm}$ ) without respiratory fluctuation and systolic pulmonary artery pressure was estimated at about $45 \mathrm{~mm} \mathrm{Hg}$. Therefore, right ventricular failure seemed to be responsible for patient's severe clinical status.

Subsequently, computed tomography pulmonary angiography ruled out pulmonary embolism and shortly afterwards an emergency call from Haematology Laboratory clarified the whole scenario. Patient's total blood count had revealed excessive leukocytosis (WBC: $390,000 / \mu \mathrm{L}$ ) with left shift of differentiation (Fig. 4), blasts about $2 \%$ and basophils $<20 \%$, normochromic normocytic anemia, grade II thrombocytopenia (PLTs: 70,000/ $\mu \mathrm{L}$ ) and scarce fragmented erythrocytes, which led to the diagnosis of CML in CP or AP with subsequent leukostasis syndrome. Diagnosis was further confirmed during the next day with peripheral blood FISH which revealed BCR-ABL1 mutation (Fig. 5). Biochemical tests revealed acute renal failure (urea $136 \mathrm{mg} / \mathrm{dL}$, creatinine $1.8 \mathrm{mg} / \mathrm{dL}$, and eGFR $30 \mathrm{~mL} / \mathrm{min} / 1.73 \mathrm{~m}^{2}$ ) attributed either to decreased cardiac output or to extreme uric acid elevation $(13 \mathrm{mg} / \mathrm{dL})$. Along with expected elevated LDH levels (350 U/L), significant hepatic biochemistry aggravation (SGOT 574 U/L, SGPT $85 \mathrm{U} / \mathrm{L}$, total bilirubin $1.8 \mathrm{mg} / \mathrm{dL}$, and direct bilirubin $1.5 \mathrm{mg} /$ $\mathrm{dL}$ ) was noticed, attributed either to hepatic congestion due to decompensated right heart failure, or to hepatic infiltration by myeloid precursors. In parallel, tumor lysis syndrome was suspected because of simultaneous hypocalcemia, elevated levels of $\mathrm{P}$, hyperuricemia and severe hyperkalemia $\left(\mathrm{K}^{+} 7.7 \mathrm{mmol} / \mathrm{L}\right)$, although acute renal failure could also explain these markers. Hyperkalemia could also be the effect of severe acidosis. Pleural puncture was carried out, with pleural fluid being characterized as exudate. Besides, there was a significant increase in inflammatory markers (CRP $184 \mathrm{mg} / \mathrm{L}$ and procalcitonine $18 \mathrm{ng} / \mathrm{mL}$ ), although no pathogen was isolated or cultured from blood, pleural fluid and urine. Coagulation monitoring showed spontaneous prolongation of PT (23") and aPTT (41"), which was attributed either to subclinical diffuse intravascular coagulation (DIC) due to tumor lysis syndrome (which could

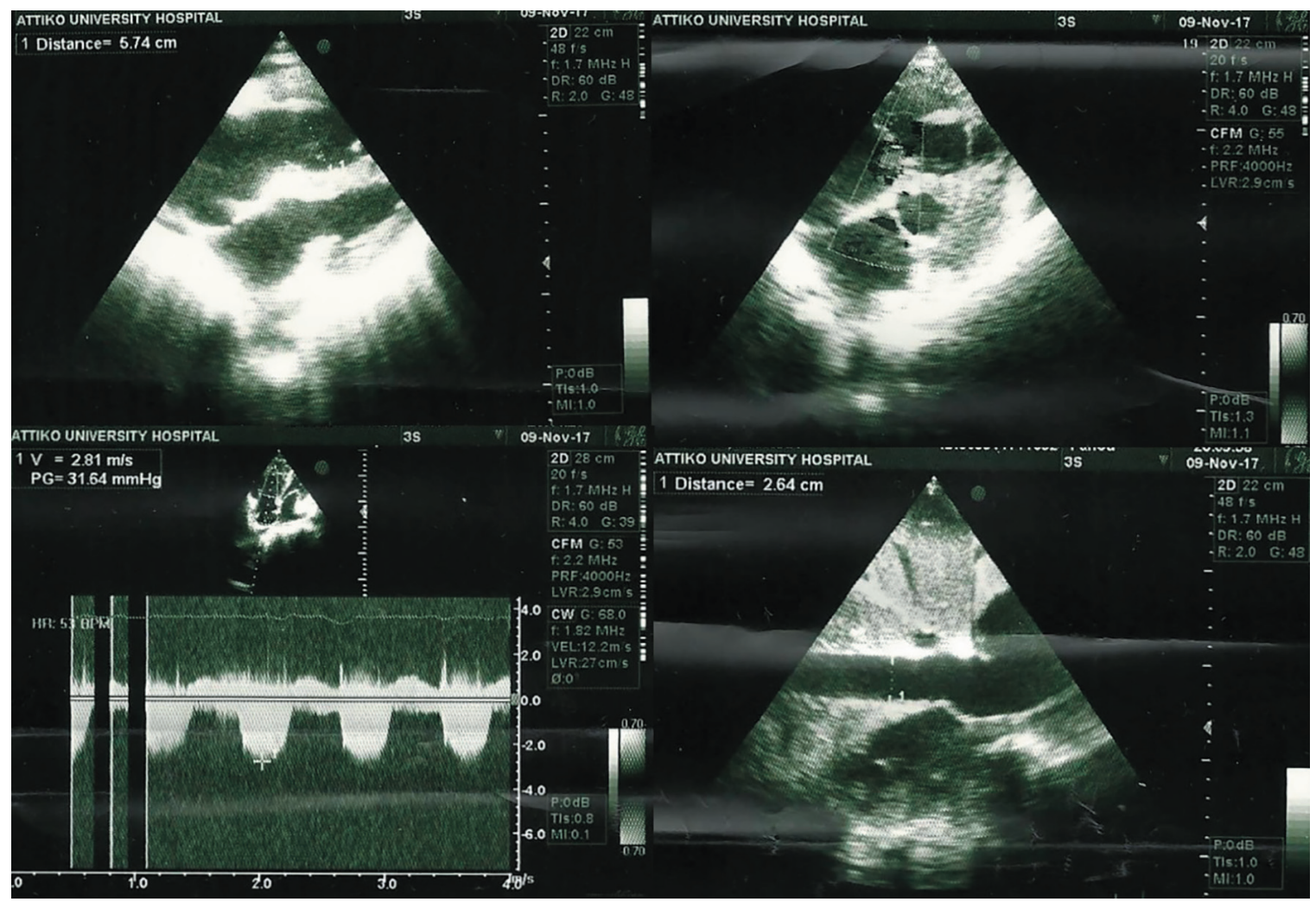

Figure 3. Echocardiogram with dilated right ventricle and pulmonary hypertension. 


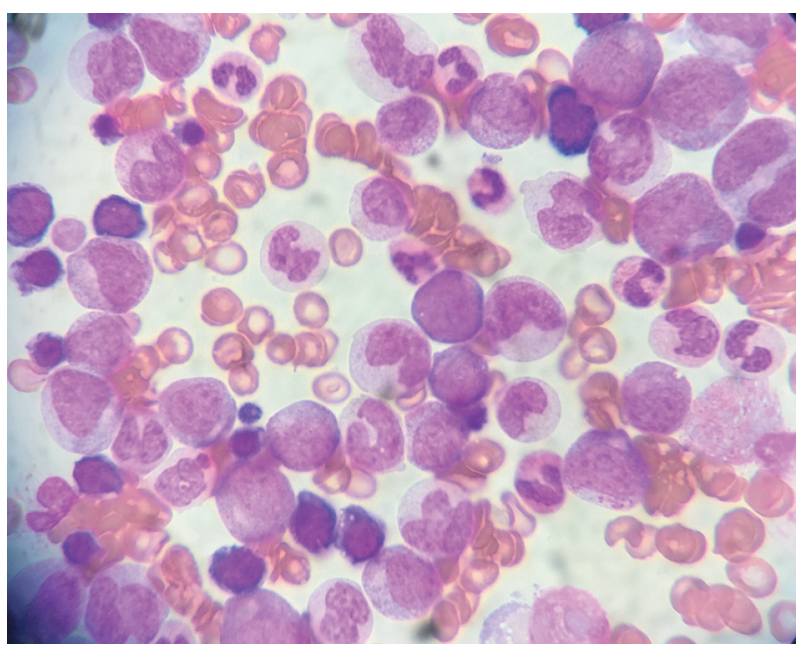

Figure 4. Patient's peripheral blood smear, with marked leukocytosis and left shift of differentiation.

also explain moderate thrombocytopenia) or to aggravate liver function. Increased D-dimers $(3,732 \mathrm{ng} / \mathrm{mL})$ also advocated the former suggestion, despite normal fibrinogen values.

Afterwards, the patient was led to the ICU and as she was not suitable for immediate leukapheresis, continuous intravenous infusion of $200 \mathrm{mg}$ Ara-C was started, in order to reduce WBC count, aiming to administer a second generation TKI per os after clinical improvement. Unfortunately, patient's clinical status continued to deteriorate and an hour after the ICU admission circulatory collapse occurred. Resuscitation procedure started immediately and pulseless electrical activity was noticed. Despite our efforts towards circulation restorage, patient passed away almost $2 \mathrm{~h}$ after admitting to the emergency department.

\section{Discussion}

Hyperleukocytosis refers to a laboratory abnormality that has been variably defined as a total WBC count greater than $100,000 / \mu \mathrm{L}$ and seen in $5-20 \%$ of patients with acute leukemias. Leukostasis, which constitutes the "symptomatic" spectrum of hyperleukocytosis, is an urgent medical situation most commonly seen in patients with acute leukemia or CML in blast crisis. It is characterized by an extremely elevated blast cell count and symptoms of decreased tissue perfusion. The pathophysiology of the syndrome is mainly derived from excessive white cell plug formation in the microvasculature. Blast cell aggregates can cause vascular occlusion, leading to ischemic tissue injury. In parallel, blasts have a higher rate of oxygen consumption and thus may compete with tissue cells in areas of obstructed flow, whereas they secrete cytokines (TNF- $\alpha$ and interleukin-1 $\beta$ ) that increase endothelial cell activation and induce adhesion receptors on blast cells themselves, promoting their adhesion to the vascular endothelium. Complement induced granulocyte aggregation is another well-documented mechanism, along with overexpression of endothelial cell adhesion molecules, such as CD54, CD62E, CD62P and CD106.

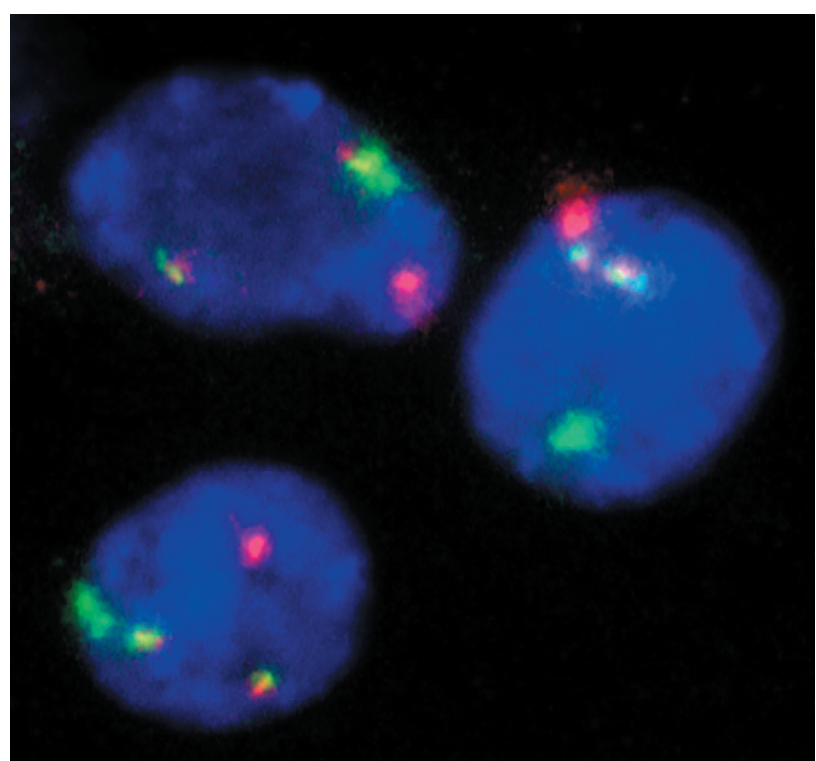

Figure 5. Interphase nuclei from peripheral blood smear positive for $\mathrm{BCR} / \mathrm{ABL} 1$ rearrangement (fusion signals).

Clinically, leukostasis is typically diagnosed empirically when a patient with leukemia and hyperleukocytosis presents with respiratory or neurological distress. Symptoms are related to decreased tissue perfusion and the most commonly seen are pulmonary related (dyspnea, tachypnea and hypoxia) and central nervous system related symptoms (visual changes, headache, dizziness, tinnitus, gait instability, confusion, somnolence and occasionally coma). Less common findings include neck vein distension, gallop rhythm and electrocardiographic signs of right ventricular overload, myocardial ischemia, priapism, acute limb ischemia, bowel infarctions, renal vein thrombosis, sudden deafness or vision loss and avascular bone necrosis. Proper treatment (leukapheresis, cytoreductive chemotherapy, and supportive care) is immediately indicated, because if left untreated, the syndrome's 1-week mortality rate varies from $20 \%$ to $40 \%$. In general, symptoms of leukostasis are more common in leukemias with large, poorly deformable blasts, such as acute myeloid or lymphoblastic leukemia, especially when total WBC count exceeds $100,000 / \mu \mathrm{L}$ and/or blast cells exceed $30,000 / \mu \mathrm{L}$. Leukostasis is rare in both CML (in CP or AP) and CLL. In CML patients, leukostasis occurs more often in blast crisis or when total WBC count exceeds 300,000/ $\mu \mathrm{L}[6,7]$.

According to literature, leukostasis in CML and other granulocytic leukemias has been reported so far from several groups. Thompson et al reported a fatal case of a young man with CML in AP who presented with sudden vision loss and subarachnoid hemorrhage [8], while Bloom et al reported a fatal case of a 49-year-old man with CML in blast crisis who developed respiratory failure [9]. Vernant et al reported 25 cases of hyperleukocytic granulocytic leukemias complicated with acute respiratory distress resolved with leukapheresis and cytoreduction [10]. The authors speculated that greater deformability of the circulating cells in CP of the above leukemias explains the lower incidence of leukostasis. Dhand et 
al [11] and Goenka et al [12] reported two cases of CML patients with pulmonary leukostasis, the one of them being in accelerated phase of the disease. Frost et al report a case of respiratory distress in a patient with chronic granulocytic leukemia in metamorphosis and leukostasis [13], while Karp et al reported two patients with respiratory failure due to chronic granulocytic leukemia derived leukostasis, which resolved with leukapheresis [14]. The effective role of leukapheresis in pulmonary leukostasis resolvement in CML was also confirmed by different groups [15-18]. Rowe et al reported that patients under 20 years of age with newly diagnosed CML had significantly increased WBC count compared to newly diagnosed CML patients over 20 years of age (average $360,000 / \mu \mathrm{L}$ vs. average $137,000 / \mu \mathrm{L}$ respectively), suggesting that there is a decrease in the average leukocyte count at presentation with increasing age in CML patients [19]. In fact, leukostasis rate was about $12 \%$ in newly diagnosed adult CML patients, compared to almost $60 \%$ in newly diagnosed CML patients under 20 years old. Sharma et al suggested that hydroxyurea could be effective in lessening blood viscosity in CML derived hyperleukocytosis [20] and Leis et al more recently confirmed the effective role of Glivec in pulmonary leukostasis due to CML blast crisis [21]. Rottenstreich et al reported a case of bilateral calf pain and retinal hemorrhages due to leukostasis in a young CML male [22] and Awh et al reported a case with leukostasis related retinopathy in a 41-year-old man with CML and severe hyperleukocytosis successfully treated with dasatinib [23]. Three different groups [6, 24, 25] presented three cases of femoral necrosis as initial presentation of CML with hyperleukocytosis. Tsai et al reported a sudden hearing loss as the initial manifestation of CML in a child [26], while Woldag et al reported a labyrinth apoplexy in a 45-year-old patient with CML and hyperleukocytosis [27] and Frankel et al reported a case of "acral lividosis" in a similar patient [28]. Kurosawa et al published a retrospective study of 256 young CML patients (children and adolescents), showing that leukostasis rate was about $10 \%$ during the course of the disease [29]. Additionally, Van Buchem et al proposed that leukemic cells in pulmonary leukostasis due to CML adhere to lung endothelial cells, the latter overexpressing vascular endothelial cell adhesion molecule-1 (VCAM-1), endothelial leukocyte adhesion molecule-1 (ELAM-1) and intercellular cell adhesion molecule-1 (ICAM-1) [30].

Our case bears some particular characteristics which are rarely featured. First of all, our patient was a middle-aged adult and according to literature newly diagnosed CML adults seldom present with hyperleukocytosis and leukostasis, in contrast with CML children or adolescents. In parallel, in developed countries, CML nowadays is usually diagnosed via a random complete blood count which is carried out for irrelevant reasons (e.g. annual preventive laboratory exams), thus the disease is initially diagnosed in $\mathrm{CP}$ and in otherwise asymptomatic individuals. Our patient initially presented fatal cardiopulmonary failure due to leukostasis, which had a hyperacute onset, with an only 2 -day history. Taking into account that her medical history was free from respiratory or heart diseases, her severe cardiopulmonary manifestation could only be attributed to leukostasis itself. Regarding patient's respiratory and right ventricular failure, point of care echocardiogra- phy, being a safe, precise, easy to perform and cost-effective technique [31], played a critical role in accelerating diagnostic procedure in terms of heart structure, heart function and hemodynamic profile assessment, without moving the fragile patient in another department. Furthermore, AP of the disease was not confirmed, because cytogenetic analysis that could have revealed additional - except Philadelphia chromosome cytogenetic abnormalities, was not performed. Nevertheless, patient's thrombocytopenia could have been the result of tumor lysis and DIC and no other criteria of AP-CML diagnosis were met. Taking into account that disease was diagnosed rather in $\mathrm{CP}$, and given that hyperleukocytosis and leukostasis is rare in newly diagnosed CP-CML, someone could hypothesize that our patient had suffered from CP-CML for months being completely asymptomatic and manifested acute severe respiratory distress when WBC outreached 300,000/ $\mu \mathrm{L}$. Additionally, her blast count and proportion in peripheral blood was not remarkably increased, a fact which is contradicting with published data which confirm the relationship between leukostasis severity and blast count in CML, along with the increased leukostasis rate in blastic transformation of the disease. Furthermore, our patient was under medical follow-up for major depression during the last year, but unluckily nobody prescribed her a complete blood count before or after depression diagnosis, that could have revealed CML in asymptomatic stage. Unfortunately, patient was not suitable for leukapheresis and despite our efforts in terms of supportive care and cytoreductive chemotherapy, she passed away before molecular or cytogenetic confirmation of CML, which was made during the next few hours.

\section{References}

1. Soverini S, De Benedittis C, Mancini M, Martinelli G. Best practices in chronic myeloid leukemia monitoring and management. Oncologist. 2016;21(5):626-633.

2. Perrotti D, Jamieson C, Goldman J, Skorski T. Chronic myeloid leukemia: mechanisms of blastic transformation. J Clin Invest. 2010;120(7):2254-2264.

3. Jabbour E, Kantarjian H. Chronic myeloid leukemia: 2014 update on diagnosis, monitoring, and management. Am J Hematol. 2014;89(5):547-556.

4. Mughal TI, Radich JP, Deininger MW, Apperley JF, Hughes TP, Harrison CJ, Gambacorti-Passerini C, et al. Chronic myeloid leukemia: reminiscences and dreams. Haematologica. 2016;101(5):541-558.

5. Bumbea H, Vladareanu AM, Voican I, Cisleanu D, Barsan L, Onisai M. Chronic myeloid leukemia therapy in the era of tyrosine kinase inhibitors - the first molecular targeted treatment. J Med Life. 2010;3(2):162-166.

6. Moon JY, Kim BS, Yun HR, Choi JH, Lee YY, Kim IS, Ahn MJ. A case of avascular necrosis of the femoral head as initial presentation of chronic myelogenous leukemia. Korean J Intern Med. 2005;20(3):255-259.

7. Aqui N, O'Doherty U. Leukocytapheresis for the treatment of hyperleukocytosis secondary to acute leukemia. Hematology Am Soc Hematol Educ Program. 2014;2014(1):457-460. 
8. Thompson DS, Goldstone AH, Parry HF, Richards JD. Leukostasis in chronic myeloid leukaemia. Br Med J. 1978;2(6131):202.

9. Bloom R, Taveira Da Silva AM, Bracey A. Reversible respiratory failure due to intravascular leukostasis in chronic myelogenous leukemia. Relationship of oxygen transfer to leukocyte count. Am J Med. 1979;67(4):679683.

10. Vernant JP, Brun B, Mannoni P, Dreyfus B. Respiratory distress of hyperleukocytic granulocytic leukemias. Cancer. 1979;44(1):264-268.

11. Dhand R, Gupta A, Deodhar SD. Pulmonary leukostasis in a patient with chronic myeloid leukemia. Indian J Chest Dis Allied Sci. 1986;28(4):253-256.

12. Goenka P, Chait M, Hitti IF, Glasberg SS, Narasimhan P. Acute leukostasis pulmonary distress syndrome. J Fam Pract. 1992;35(4):445-446, 449.

13. Frost T, Isbister JP, Ravich RB. Respiratory failure due to leukostasis in leukaemia. Med J Aust. 1981;2(2):94-95.

14. Karp DD, Beck JR, Cornell CJ, Jr. Chronic granulocyte leukemia with respiratory distress. Efficacy of emergency leukapheresis. Arch Intern Med. 1981;141(10):13531354.

15. Bubala H, Sonta-Jakimczyk D, Janik-Moszant A, Szczepanski T, Tomaszewska R, Drybanska B, Sierantowicz-Zdaniewicz U, et al. [Leukapheresis in children with chronic myeloid leukemia and pulmonary leukostasis]. Pol Merkur Lekarski. 2004;17(101):500-502.

16. Veljkovic D, Kuzmanovic M, Micic D, Serbic-Nonkovic O. Leukapheresis in management hyperleucocytosis induced complications in two pediatric patients with chronic myelogenous leukemia. Transfus Apher Sci. 2012;46(3):263-267.

17. Yoshida M, Ueda H, Imaizumi J, Horiuchi M, Hagihara K, Kanashima H, Nakao T, et al. [Leukostasis during chronic myelogenous leukemia resolved following leukapheresis]. Gan To Kagaku Ryoho. 2015;42(6):763-765.

18. Shafique S, Bona R, Kaplan AA. A case report of therapeutic leukapheresis in an adult with chronic myelogenous leukemia presenting with hyperleukocytosis and leukostasis. Ther Apher Dial. 2007;11(2):146-149.

19. Rowe JM, Lichtman MA. Hyperleukocytosis and leukostasis: common features of childhood chronic myelogenous leukemia. Blood. 1984;63(5):1230-1234.

20. Sharma K, Rao S, Bhat SV. Effect of hydroxyurea on blood viscosity in chronic myelogenous leukemia with hyperleukocytosis. Physiol Chem Phys Med NMR. 1991;23(4):261-268.

21. Leis JF, Primack SL, Schubach SE, Curtin PT, Druker BJ, Maziarz RT. Management of life-threatening pulmonary leukostasis with single agent imatinib mesylate during CML myeloid blast crisis. Haematologica. 2004;89(9):ECR30.

22. Rottenstreich M, Lavie M, Rottenstreich A. [Chronic myeloid leukemia in a young male presenting with bilateral calf pain due to leukostasis]. Harefuah. 2015;154(5):296298, 340 .

23. Awh CC, Miller JB, Wu DM, Eliott D. Leukostasis Retinopathy: A new clinical manifestation of chronic myeloid leukemia with severe hyperleukocytosis. Ophthalmic Surg Lasers Imaging Retina. 2015;46(7):768-770.

24. Kraemer M, Weissinger F, Kraus R, Beer M, Kunzmann V, Wilhelm M. Aseptic necrosis of both femoral heads as first symptom of chronic myelogenous leukemia. Ann Hematol. 2003;82(1):44-46.

25. Kumar S, Bansal D, Prakash M, Sharma P. Avascular necrosis of femoral head as the initial manifestation of CML. Pediatr Hematol Oncol. 2014;31(6):568-573.

26. Tsai CC, Huang CB, Sheen JM, Wei HH, Hsiao CC. Sudden hearing loss as the initial manifestation of chronic myeloid leukemia in a child. Chang Gung Med J. 2004;27(8):629-633.

27. Woldag K, Schwarzer A. [Chronic myeloid leukemia - a rare cause of labyrinth apoplexy]. Laryngorhinootologie. 1996;75(4):247-249.

28. Frankel DH, Larson RA, Lorincz AL. Acral lividosis - a sign of myeloproliferative diseases. Hyperleukocytosis syndrome in chronic myelogenous leukemia. Arch Dermatol. 1987;123(7):921-924.

29. Kurosawa H, Tanizawa A, Tono C, Watanabe A, Shima H, Ito M, Yuza Y, et al. Leukostasis in children and adolescents with chronic myeloid leukemia: Japanese Pediatric Leukemia/Lymphoma Study Group. Pediatr Blood Cancer. 2016;63(3):406-411.

30. van Buchem MA, Hogendoorn PC, Bruijn JA, Kluin PM. Endothelial activation antigens in pulmonary leukostasis in leukemia. Acta Haematol. 1993;90(1):29-33.

31. Neskovic AN, Hagendorff A, Lancellotti P, Guarracino F, Varga A, Cosyns B, Flachskampf FA, et al. Emergency echocardiography: the European Association of Cardiovascular Imaging recommendations. Eur Heart J Cardiovasc Imaging. 2013;14(1):1-11. 